\title{
Electron Cyclotron Heating Start-Up Experiments on TST-2
}

\author{
Junichi SUGIYAMA, Akira EJIRI, Yuichi TAKASE, Osamu WATANABE ${ }^{2)}$, Yuuki ADACHI, \\ Hiroshi TOJO, Makoto SASAKI ${ }^{1)}$, Tetsuya MASUDA, Takuya OOSAKO and Soichiro KAINAGA ${ }^{1)}$ \\ Graduate School of Frontier Sciences, The University of Tokyo, Kashiwa 277-8561, Japan \\ ${ }^{1)}$ Graduate School of Science, The University of Tokyo, Bunkyo 113-0033, Japan \\ ${ }^{2)}$ High Temperature Plasma Center, The University of Tokyo, Kashiwa 277-8561, Japan
}

(Received 7 January 2008 / Accepted 7 April 2008)

\begin{abstract}
Experiments were performed to study non-inductive current generation by electron cyclotron heating $(\mathrm{ECH})$ in the TST-2 spherical tokamak. A magnetron $(2.45 \mathrm{GHz} / 5 \mathrm{~kW})$ and a horn antenna were used to inject either the $\mathrm{O}$ - or X-mode. The maximum plasma current does not depend on the injected wave polarization; however, it has a weak dependence on the vertical field configuration and is proportional to the vertical field. The initial current ramp-up rate depends on various operational parameters. The ramp-up rate increases with the injected EC wave power, and decreases with the filling pressure, resonance position (i.e., the toroidal field strength), and vertical field strength. It also depends on the magnetic field configuration. Conversely, the ramp-up rate does not depend on wave polarization, suggesting that multiple pass absorption of the EC wave is important.
\end{abstract}

(c) 2008 The Japan Society of Plasma Science and Nuclear Fusion Research

Keywords: spherical tokamak, electron cyclotron heating, current drive, plasma start-up

DOI: $10.1585 /$ pfr.3.026

A key issue in spherical tokamak (ST) research is to develop a method for initiating the plasma current and forming an ST configuration without using the central Ohmic solenoid. Various scenarios have been studied on ST devices. Electron cyclotron heating $(\mathrm{ECH})$ start-up is one such method, and many experiments on ST devices have been carried out [1-13], but the current drive mechanism is still not clearly identified. Moreover, the mechanism of current jump, which is believed to occur when closed flux surfaces are formed, is not completely understood. A major difficulty in these studies is that the power deposition profile and the phase space region of waveparticle (electron) coupling are unknown. In such a situation, experimental comparison of different injection scenarios (i.e., O-mode vs. X-mode) and various parameter dependences are crucial. There is no systematic experimental comparison between $\mathrm{O}$-mode and X-mode polarizations. The $\mathrm{X}$-mode is expected to be more effective than the O-mode, because X-mode absorption is efficient even in low density plasmas and can be converted to the electron Bernstein wave (EBW), which can heat the plasma even at densities higher than the cutoff density.

The O-mode is absorbed most effectively at the fundamental resonance, while the $\mathrm{X}$-mode is absorbed most strongly at the second harmonic resonance [14]. Therefore, these two polarizations would have different heating efficiencies and deposition profiles. Since the densities obtained in the ST experiments have the same order of magnitude as the O-mode or X-mode cutoff density, wave reflection and refraction during propagation can significantly

author's e-mail: sugiyama@fusion.k.u-tokyo.ac.jp affect the overall efficiency [5]. We also compared several vertical field configurations with different curvatures. At the beginning of a discharge, the plasma current is very low, and the particle orbits in the externally applied field are believed to play important roles in generating the initial small plasma current [15]. This indicates that the vertical field configuration would affect the formation of the initial current. A typical ECH start-up discharge can be divided into three phases: (i) the plasma production and initial slow current ramp-up phase, (ii) the rapid current ramp-up phase (i.e. current jump) $[3,17]$, and (iii) the current sustainment phase. For understanding the physics in each phase, it is important to understand the plasma response to various parameters. We performed a systematic survey of operational parameters, including wave polarization and magnetic configuration. In this paper, the parameter dependences of the initial current ramp-up rate and the sustained current, as well as the condition for current jump, are reported.

Experiments described in this paper were carried out on TST-2 [16]. Four magnetic configurations shown in Fig. 1, with different vertical field curvatures generated by different poloidal field (PF) coil sets, were compared. The radial profiles of $B_{z}$ are also shown at the bottom, and the decay index $n=-\partial \ln B_{z} / \partial \ln R$ at $R=0.38 \mathrm{~m}$ is indicated in each figure. The PF1 configuration has a strongly positive curvature (i.e., large mirror ratio), PF2 and PF1+PF2 configurations have moderately positive curvatures, and PF3 configuration has almost no curvature in most of the volume inside the vacuum vessel. The PF coil waveform is preprogrammed to provide a stationary vertical field. The electron cyclotron wave (ECW) is injected along the ma- 

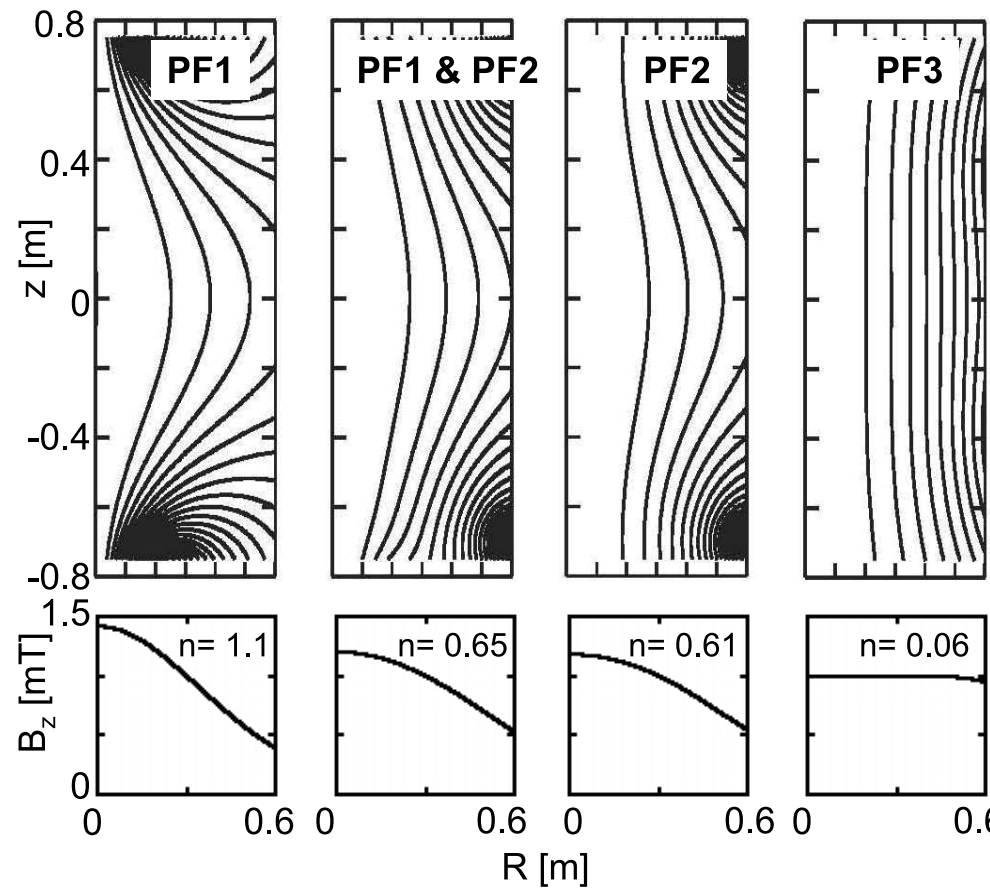

Fig. 1 Equi-flux contours for magnetic configurations and $B_{z}$ profile at $z=0$ formed by different PF coil sets. Decay index $n$ for $R=0.38 \mathrm{~m}$ is shown in the bottom figures.

jor radius (i.e., perpendicular to the magnetic field) from a port located $250 \mathrm{~mm}$ below the midplane.

The port has a quartz vacuum window, and a tapered transition from a rectangular standard waveguide to a circular aperture with a diameter $(D)$ of $0.16 \mathrm{~m}$ is used as a horn antenna to launch the wave. The wavelength $(\lambda)$ is $0.12 \mathrm{~m}$, and the beam divergence angle is about $\lambda / D=$ 0.75 . Thus, $N_{\|}$can be of order one, although the wave is injected along the major radius. By replacing a straight waveguide section with a twisted waveguide section, we can switch the wave polarization from the O-mode to the $\mathrm{X}$-mode. To monitor wave absorption and polarization, a microwave detector attached to the end of a standard waveguide section (leakage monitor) is located just outside a large quartz window, which is separated toroidally from the ECH injection port by 120 degrees. By rotating the waveguide, we can measure the O-mode (i.e., horizontally polarized) component or the X-mode (i.e., vertically polarized) components.

When we compared the $\mathrm{O}$ - and $\mathrm{X}$-mode polarizations, other parameters were kept fixed. A stationary vertical field is maintained by the PF coils, while the toroidal field (TF) coil current decays slowly, moving the fundamental resonance layer out of the vacuum vessel in $50-100 \mathrm{~ms}$. In most cases, the plasma vanishes when the fundamental resonance layer disappears from the region that can be occupied by the plasma. Figure 2 shows two almost identical discharges with $\mathrm{O}$-mode injection and $\mathrm{X}$-mode injections. After the initial gradual increase, the plasma current often shows an abrupt increase (at $40 \mathrm{~ms}$ in Fig. 2),

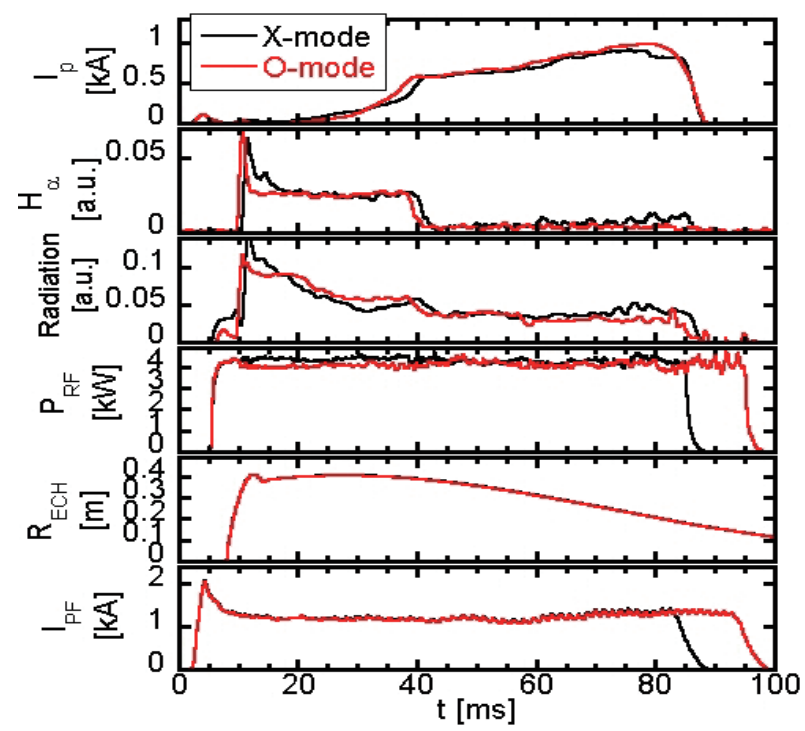

Fig. 2 Comparison of waveforms for discharges with O-mode (red) injection and X-mode (black) injection.

referred to as the current jump [3,17], after which it tends to stay nearly constant. The plasma current after the current jump is compared in different magnetic configurations as shown in Fig. 1. We found that the current is proportional to the vertical field strength $\left(B_{z}\right)$. The differences between the different magnetic configurations are not significant (Fig. 3). Compared to the PF1+PF2 configurations, the PF3 configuration shows higher currents and the PF1 


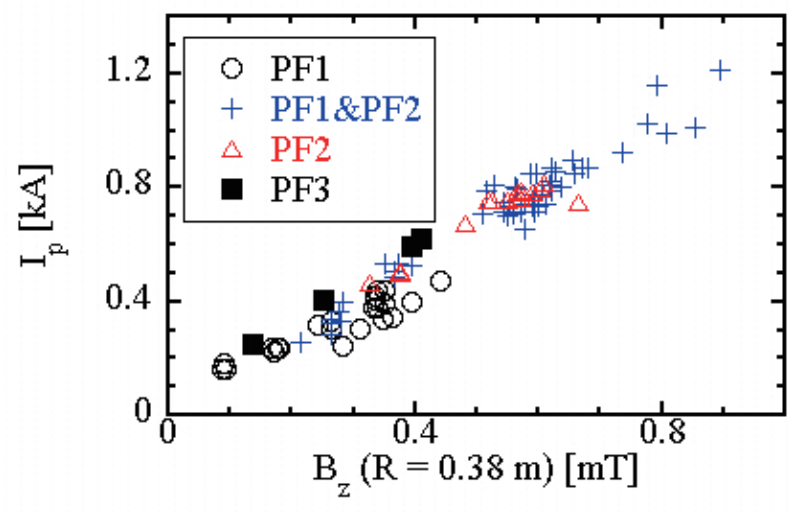

Fig. 3 Maximum plasma current after the current jump as a function of $B_{z}$ for four different vertical field configurations shown in Fig. 1: PF1 (circles), PF1+PF2 (pluses), PF2 (triangles) and PF3 (squares).

configuration shows lower currents. This difference might be related to the difference in the vertical field curvature. In equilibrium, the hoop force should be balanced by the inward force exerted by the external vertical field. Therefore, it is reasonable to observe the linear dependence on $B_{z}$. The coefficient is not inconsistent with that expected for equilibrium. The plasma current of $1 \mathrm{kA}$ for the vertical field strength of $1 \mathrm{mT}$ (see Fig. 3) can be derived from the relationship:

$$
B_{z}=\frac{\mu_{0} I_{\mathrm{p}}}{4 \pi R}\left(\ln \frac{8 \mathrm{R}}{a}+\beta_{\mathrm{p}}+\frac{l_{\mathrm{i}}}{2}-\frac{3}{2}\right),
$$

where we assume $R=0.38 \mathrm{~m}, a=0.25 \mathrm{~m}, \beta_{\mathrm{p}}+l_{\mathrm{i}} / 2=2.8$. However, the equilibrium field also depends on other parameters such as the pressure, size, and vertical field curvature. The reason for the weak dependence on the vertical field configuration is not understood. Such a stiff dependence might arise from a self-organization process, and should be further investigated. It should be noted that the coefficients (i.e., the ratio of $I_{\mathrm{p}}$ to $B_{z}$ ) are similar to those obtained in CDX-U[1], LATE [10, 11], and CPD [13], but is about 2.5 times lower than TST-2@K (TST-2 at Kyushu University) [5].

Figure 4 shows the relationship between the vertical field strength and the plasma current just before the current jump. The current jump occurs when the plasma current reaches a value proportional to the applied vertical field strength. The ratio is about $0.5 \mathrm{kA} / 1 \mathrm{mT}$ in the figure. This ratio corresponds to the condition $B_{z}=B_{\mathrm{p}}$, in which $B_{\mathrm{p}}$ is the induced poloidal field calculated by $B_{\mathrm{p}}=\mu_{0} I_{\mathrm{p}} /(2 \pi a)$ with $a=0.1 \mathrm{~m}$. When the current satisfies this condition, $B_{\mathrm{p}}$ induced by the plasma at the inboard side of the plasma boundary cancels the externally applied vertical field of strength $B_{z}$, and creates a field reversal. Thus, a close flux surface with a minor radius $a$ is created. Normally, this condition depends on the current profile. The same condition is satisfied with a rectangular cross-sectional current

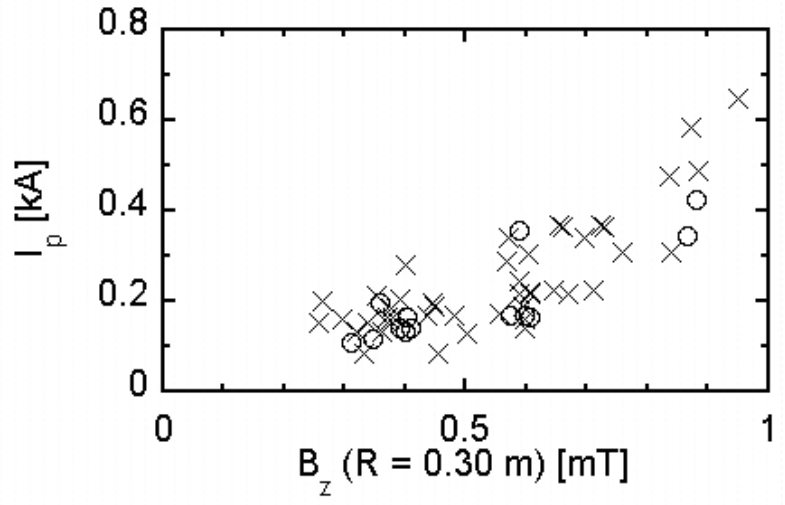

Fig. 4 Plasma current just before the current jump as a function of the applied vertical field strength $B_{z}$. Both O-mode injection (circles) and X-mode injection (crosses) cases are plotted.

channel with a width of $0.05 \mathrm{~m}$ and a height of $0.3 \mathrm{~m}$. Once the closed flux surfaces are formed, the confined orbit region in the phase space expands, resulting in an abrupt increase in the current. Besides the dependence on the vertical field strength, no dependence on other parameters such as injected EC polarizations was found. To induce current jump at an appropriate time, it is necessary to increase the plasma current at an appropriate ramp-up rate, since the available discharge duration is limited by the slowly decaying toroidal field (Fig. 2). Thus, we need to understand the dependence of the ramp-up rate on the operational conditions. Evaluation of the current ramp-up rate is also important for extrapolating of the present results in small STs to larger STs, where appropriate currents with certain magnetic and kinetic energies should be generated with a limited heating power. We investigate the dependences on the incident power, filling pressures, and vertical field. Figure 5 shows the relationship between the incident power and the ramp-up rate. Higher power results in a faster ramp-up rate. No significant difference is observed between the $\mathrm{O}$-mode and $\mathrm{X}$-mode polarizations.

Figure 6 (a) shows the current ramp-up rate as a function of RF power for different TF strengths. A lower TF strength is favorable for faster ramp-up, presumably because the EC resonance layer is located at a smaller major radius. Figures 6 (b) and (c) show the dependences on the $B_{z}$ strength and the filling pressure. Faster ramp-up rates are obtained at lower filling pressures and lower vertical field strengths. At a lower TF strength, the injected power is deposited in a smaller volume because of the smaller resonance radius. As a result, electrons can acquire higher energies. Higher energy electrons contribute to the plasma current more efficiently compared to lower energy electrons. The importance of high-energy electrons is also suggested based on the experimental results from LATE [18]. Lower filling pressures result in a similar situation, favor- 


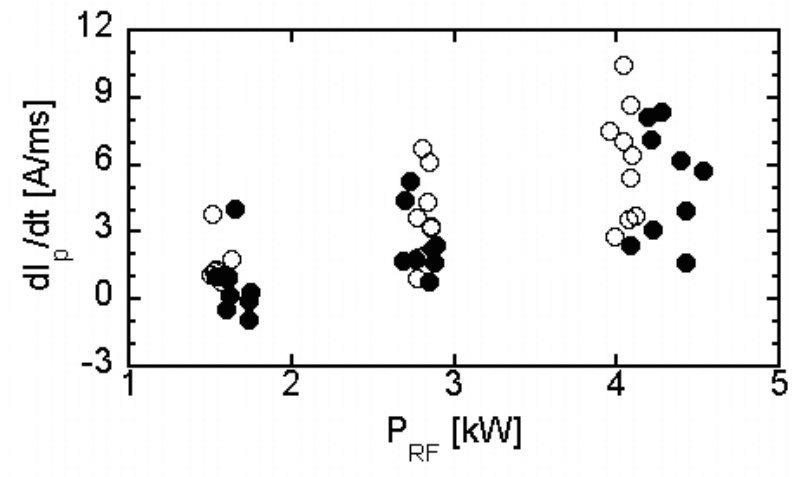

Fig. 5 Dependence of the current ramp-up rate on RF power for $\mathrm{O}$-mode injection (filled circles) and $\mathrm{X}$-mode injection (open circles).
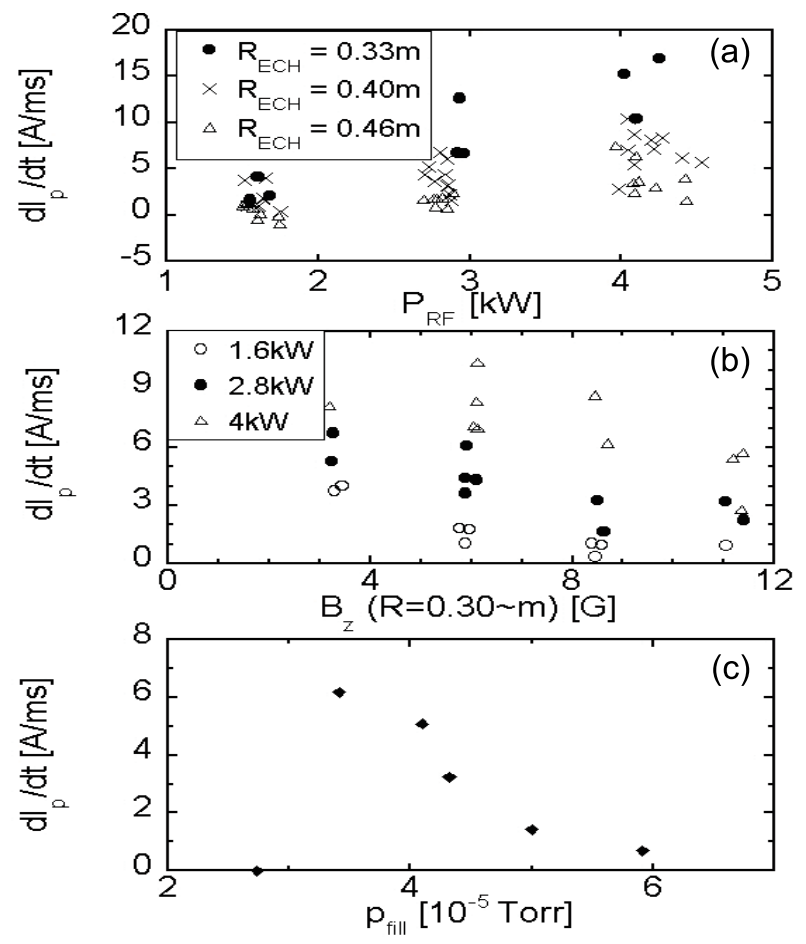

Fig. 6 Dependences of current ramp-up rate on (a) RF power, (b) $B_{z}$ strength, and (c) filling pressure.

ing the formation of higher energy electrons. Furthermore, the lower electron-neutral particle collision frequency reduces both momentum and particle losses. A lower vertical field strength is favorable because of the larger perpendicular drift and the larger contribution to the toroidal current by each electron [15].

As shown in Fig. 7, the experimentally observed current ramp-up rate before the current jump appears to scale as

$$
\frac{\mathrm{d} I_{\mathrm{p}}}{\mathrm{d} t}=C_{\mathrm{PF}} \times \frac{P_{\mathrm{RF}}^{2.1 \pm 0.1}}{R_{\mathrm{ECH}}^{2.4_{-0.4}^{+0.2}} p_{\text {fill }}^{1.0 \pm 0.1} B_{z}^{0.4_{-0.1}^{+0.4}}},
$$

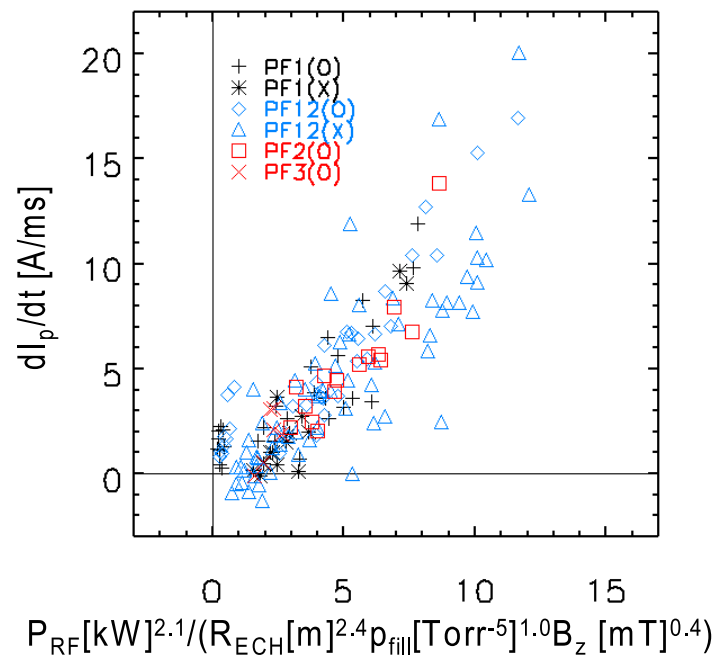

Fig. 7 Empirical power law scaling of current ramp-up rate.

where, $R_{\mathrm{ECH}}$ represents the major radius of the $\mathrm{ECH}$ fundamental resonance, $B_{z}$ represents the vertical field strength at the same $R$, and $P_{\mathrm{RF}}$ represents the injected RF power. The current ramp-up rate also depends on the PF configuration (shown by different symbols in Fig. 7). $C_{\mathrm{PF}}$ is a coefficient that depends on the vertical field configuration, and the relative magnitudes for the PF1, PF12, PF2, and PF3 configurations are in the ratio $1: 1.7: 1.4: 0.8$. Among the four PF configurations, PF1+PF2 configuration with moderate positive curvature exhibits the highest current ramp-up rates, $\mathrm{PF} 1$ with the strongest curvature shows smaller ramp-up rates, while the PF3 configuration with almost no curvature shows the lowest ramp-up rates. In spite of the very large difference in the ramp-up rate, the final current is almost same (Fig. 3). These results suggest that the high-energy electron orbit plays an important role during the initial current ramp-up phase, but not after the current jump.

It is important to understand the dynamics of the current jump to clarify the formation mechanism of closed flux surfaces. Figure 8 shows waveforms for a discharge with an abrupt current jump. In this case, the electron density on the outboard side and the $\mathrm{H} \alpha$ emission show a clear increase and decrease during the current jump. According to CCD camera images, the plasma often shows a slight radial and vertical expansion during a current jump. In other cases the plasma does not show such an expansion; however it shows a gradual electron density increase and a periodical ejection of plasma from the fundamental resonance layer. Therefore, the behaviors before and during the jump are not always the same. Conversely, the plasma always shrinks vertically at a current jump.

The O-mode and $\mathrm{X}$-mode polarizations show very 


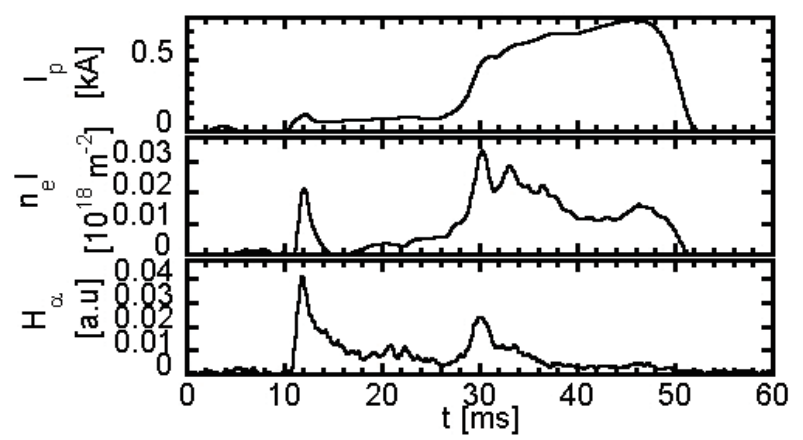

Fig. 8 Time evolution of a discharge with a current jump.

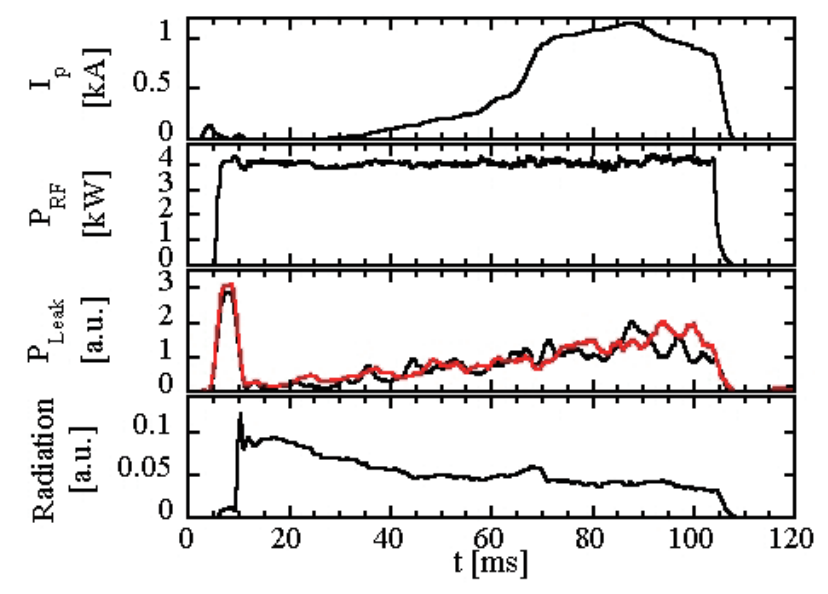

Fig. 9 Leakage O-mode (red) and X-mode (black) wave power in two similar discharges with O-mode injection.

similar behaviors not only in the achieved plasma current but also in discharge waveforms and parameter dependences. Figure 9 shows the signal detected by the leakage monitor during O-mode injection. Before plasma generation (breakdown) at $10 \mathrm{~ms}$, the O-mode leakage power is nearly the same as the X-mode leakage power. It can be understood that the injected electromagnetic wave undergoes multiple reflections inside the vessel and the polarization is randomized. When the plasma is generated, the leakage power decreases significantly, implying that the plasma absorbs most of the RF power after multiple reflections. It suggests that the polarization is randomized in a few reflections. The leakage power increases gradually and reaches a stationary level after the current jump at about half the prebreakdown level. Conversely, the radiated power from the plasma decreases with the increase in plasma current. The fact that both $\mathrm{O}$-mode and $\mathrm{X}$-mode leakage powers show similar behaviors, indicates that the wave polarization is randomized even in the presence of the plasma. These results are consistent with the fact that almost no difference is observed between the two polarizations (Figs. 2, 4, and 5).

In summary, ECH start-up experiments were performed and various parameter and configuration dependences were studied. The start-up consists of three phases: the initial current formation phase, the current jump phase, and the current sustainment phase. A scaling law for the initial current ramp-up rate was obtained. Different vertical field configurations were found to be described by different coefficients. Contrastingly, the sustained current level has no significant dependence on the vertical field configuration. In both phases, no difference was found between the different polarizations. The current jump occurs when the current reaches a value determined by the vertical field strength.

This work was supported by the Japan Society for the Promotion of Science (JSPS) under Grant-in-Aid for Scientific Research No. 16106013, and NIFS Collaborative Research Program No. NIFS07KUTR021.

[1] C.B. Forest et al., Phys. Rev. Lett. 68, 3559 (1992).

[2] C.B. Forest et al., Phys. Plasmas 1, 1568 (1994).

[3] T. Maekawa et al., Nucl. Fusion 45, 1439 (2005).

[4] Y. Takase et al., Nucl.Fusion 46, S598 (2006).

[5] A. Ejiri et al., Nucl. Fusion 46, 709 (2006).

[6] A. Ejiri et al., Trans. Fusion Sci. Tech. 51, No.2T, 168 (2007).

[7] V. Shevchenko et al., AIP Conf. Proc. 933, 323 (2007).

[8] V. Shevchenko et al., Fusion Sci. Tech. 52, 202 (2007).

[9] B. Lloyd et al., The 13th International Workshop on Spherical Torus 200710 - 12 Oct 2007, Fukuoka, Japan. http://www.triam.kyushu-u.ac.jp/ISTW2007/agenda.html

[10] H. Tanaka et al., IEEJ Trans. 125, 919 (2005).

[11] H. Tanaka et al., The 13th International Workshop on Spherical Torus 200710 - 12 Oct 2007, Fukuoka, Japan. http://www.triam.kyushu-u.ac.jp/ISTW2007/agenda.html

[12] A. Ejiri et al., The 13th International Workshop on Spherical Torus 200710 - 12 Oct 2007, Fukuoka, Japan. http://www.triam.kyushu-u.ac.jp/ISTW2007/agenda.html

[13] T. Yoshinaga et al., The 13th International Workshop on Spherical Torus 200710 - 12 Oct 2007, Fukuoka, Japan. http://www.triam.kyushu-u.ac.jp/ISTW2007/agenda.html

[14] M. Bornatici et al., Nucl. Fusion 23, 1153 (1983).

[15] A. Ejiri et al., Nucl. Fusion 47, 403 (2007).

[16] Y. Takase et al., Nucl. Fusion 41, 1543 (2001).

[17] T. Yoshinaga et al., J. Plasma Fusion Res. 81, 333 (2005).

[18] T. Yoshinaga et al., Nucl. Fusion 47, 210 (2007). 